\title{
SOME GROWTH THEOREMS FOR LINEAR ORDINARY DIFFERENTIAL EQUATIONS $\left(^{(1)}\right.$
}

BY

\author{
W. S. $\operatorname{LOUD}(2)$
}

1. Introduction. If $A(t)$ is a real, bounded, piecewise continuous, square matrix for all $t \geq 0$, it is known from the work of Liapounoff $[6]\left({ }^{3}\right)$ that if $x(t)$ is any nontrivial solution vector of the system

$$
x^{\prime}=A(t) x,
$$

there exists a finite constant $\lambda_{0}$, such that if $\lambda>\lambda_{0}$ we have

$$
\lim _{t \rightarrow \infty} x(t) e^{-\lambda t}=0 .
$$

The number $\lambda_{0}$ furnishes a limitation on the growth of solutions of (1.1). In [6], $-\lambda_{0}$ is called the characteristic number of the vector solution $x(t)$.

In this paper we shall give upper bounds for $\lambda_{0}$ for several cases of (1.1). The estimates will be derived solely from bounds of $A(t)$. In the second-order scalar case, considered in $\S 4$, the results obtained will be best possible when $A(t)$ is a constant.

2. Preliminary definitions. If $A(t)=\left[a_{i j}(t)\right], i, j=1,2, \cdots, m$, is an $m \times m$ square matrix of functions, we define the absolute value of $A(t)$ by the expression

$$
|A(t)|=\sum_{i, j=1}^{m}\left|a_{i j}(t)\right| .
$$

If $x(t)=\left\{x_{i}(t)\right\}, i=1,2, \cdots, m$, is a vector of order $m$, we define the absolute value of $x(t)$ by the expression

$$
|x(t)|=\sum_{i=1}^{m}\left|x_{i}(t)\right| .
$$

We now define a set of functions related to the exponential function $e^{t}$. For all pairs of integers $(k, n), k=0, \pm 1, \pm 2, \cdots, n=1,2, \cdots$, we set

$$
\phi_{k}^{(n)}(t)=\sum_{j \equiv k(\bmod n) ; j \geqq 0} \frac{t^{j}}{j !} .
$$

Presented to the Society, August 24, 1956; received by the editors June 30, 1956.

(1) The research for this paper was supported in part by the Office of Ordnance Research, U. S. Army, Contract No. DA-11-022-ORD-1869.

(2) This paper was written while the author was a Visiting Fellow at the Massachusetts Institute of Technology.

(3) Numbers in brackets refer to the bibliography at the end of the paper. 
For any $n$, there are only $n$ distinct $\phi$ 's, since $\phi_{i}^{(n)}(t)=\phi_{j}^{(n)}(t)$ in case $i \equiv j(\bmod n)$. As examples, $\phi_{k}^{(1)}(t)=e^{t}$ for all $k, \phi_{k}^{(2)}(t)=\cosh t$ if $k$ is even, and $\phi_{k}^{(2)}(t)=\sinh t$ if $k$ is odd. The set of functions $\phi_{\boldsymbol{k}}^{(n)}(t)$ also has the following properties which are clear from the definition.

$$
\begin{aligned}
& \text { (a) For all } t \geq 0, \quad\left|\phi_{k}^{(n)}(t)\right| \leq e^{t}, \\
& \text { (b) For all } t, \sum_{k=0}^{n-1} \phi_{k}^{(n)}(t)=e^{t}, \\
& \text { (c) } \frac{d}{d t} \phi_{k}^{(n)}(t)=\phi_{k-1}^{(n)}(t) .
\end{aligned}
$$

The solution of the scalar initial value problem

$$
x^{(n)}=x \quad x^{(j)}(0)=a_{j}, \quad j=0,1, \cdots, n-1,
$$

is given by

$$
x(t)=\sum_{j=0}^{n-1} a_{j} \phi_{j}^{(n)}(t)
$$

\section{A growth theorem for a vector system.}

TheOREM 1. Let $x$ be a vector of order $m$, and let $A(t)$ be an $m \times m$ square matrix of real, bounded, piecewise continuous functions of $t$ for all $t \geq 0$. Let the absolute value of $A(t)$ satisfy $|A(t)| \leq A^{n}$ for some positive number $A$. Let $\phi(t)$ be the solution vector of

$$
x^{(n)}=A(t) x
$$

having $\phi^{(j)}(0)=a_{j}$ for $j=0,1, \cdots, n-1$. Then for all $t \geq 0$,

$$
\begin{aligned}
\text { (a) }|\phi(t)| & \leq \sum_{j=0}^{n-1}\left|a_{j}\right| A^{-i} \phi_{j}^{(n)}(A t), \\
\left|\phi^{(k)}(t)\right| & \leq \sum_{j=0}^{n-1}\left|a_{j}\right| A^{k-j} \phi_{j-k}^{(n)}(A t) \quad k=1,2, \cdots, n-1 .
\end{aligned}
$$

(b) If $K$ denotes the maximum of the $n$ quantities $A^{-j}\left|a_{j}\right|$, then for all $t \geq 0$,

$$
|\phi(t)| \leq K e^{A t} \quad\left|\phi^{(k)}(t)\right| \leq K A^{k} e^{A t}, \quad k=1,2, \cdots, n-1 .
$$

(c) For the system (3.1), $\lambda_{0} \leq A$.

Proof of Theorem 1. Part (b) is an immediate conclusion from part (a), using (b) of (2.4). Part (c) comes directly from part (b) by definition of $\lambda_{0}$.

The proof of part (a) uses a technique of Liapounoff, see [6, p. 403]. 
The equation (3.1) and the initial condition imply that $\phi(t)$ is the unique solution of the integral equation

$$
x(t)=a_{0}+a_{1} t+\cdots+a_{n-1} \frac{t^{n-1}}{(n-1) !}+\int_{0}^{t} \frac{(t-s)^{n-1}}{(n-1) !} A(s) x(s) d s .
$$

If $f_{0}(t)=\sum_{0}^{n-1} a_{j} t^{i} / j !$, and the functions $f_{p}(t)$ are defined by the relation

$$
f_{p+1}(t)=\int_{0}^{t} \frac{(t-s)^{n-1}}{(n-1) !} A(s) f_{p}(s) d s, \quad p=0,1,2, \cdots
$$

the sum $\sum_{0}^{\infty} f_{p}(t)$ formally satisfies (3.4). It is readily established by mathematical induction that

$$
\left|f_{p}(t)\right| \leq A^{p n} \sum_{j=0}^{n-1}\left|a_{j}\right| \frac{t^{p n+j}}{(p n+j) !} .
$$

This is shown using the facts that the absolute value of the sum of a set of vectors does not exceed the sum of their absolute values, that for a matrix $A$ and a vector $x,|A x| \leq|A||x|$, and that

$$
\int_{0}^{t} \frac{(t-s)^{n-1} s^{q}}{(n-1) ! q !} d s=\frac{t^{q+n}}{(q+n) !} .
$$

From (3.6) it is clear that on any finite interval $[0, t]$, the series $\sum_{0}^{\infty} f_{p}(t)$ is absolutely-uniformly convergent, so that termwise integration is allowed, and the series is actually equal to $\phi(t)$. Assertion (3.2) in the theorem is a rewriting of the statement $|\phi(t)| \leq \sum_{0}^{\infty}\left|f_{p}(t)\right|$ using (3.6) and (2.3).

If $k$ is one of $1,2, \cdots, n-1$, we find by differentiating (3.5) $k$ times that

$$
f_{p}^{(k)}(t)=\int_{0}^{t} \frac{(t-s)^{n-1-k}}{(n-1-k) !} A(s) f_{p-1}(s) d s
$$

from which, using (3.6) we find

$$
\left|f_{p}^{(k)}(t)\right| \leq A^{p n} \sum_{j=0}^{n-1}\left|a_{i}\right| \frac{t^{p n+j-k}}{(p n+j-k) !} .
$$

From (3.7) we have that on any finite interval $[0, t]$ the series $\sum_{0}^{\infty} f_{p}^{(k)}(t)$ is absolutely-uniformly convergent; and so represents the $k$ th derivative of $\phi(t)$. Assertion (3.3) of the theorem is a restatement of

$$
\left|\phi^{(k)}(t)\right| \leq \sum_{0}^{\infty}\left|f_{p}^{(k)}(t)\right|
$$

using (3.7) and (2.3). This completes the proof of Theorem 1. 
Equation (3.1) is a very special case of the $n$th order linear equation in that lower order derivatives are not present. The proof depends on this restriction, and the result is not true without it.

It is possible to write (3.1) as a first-order system of dimension $m n$, and then apply Theorem 1 . The result is not so good. The $m n \times m n$ matrix will have absolute value $m(n-1)+|A(t)| \leq m(n-1)+A^{n}$. Since the order of the system is one, the majorizing exponential will be $\exp \left[\left(A^{n}+m(n-1)\right] t\right.$, which is larger than $e^{A t}$.

4. The second-order scalar case. We now restrict ourselves to the secondorder scalar case

$$
x^{\prime \prime}+a(t) x=0,
$$

where $a(t)$ is real, piccewise continuous, and bounded. If $\alpha \leq a(t) \leq \beta$, we can apply Theorem 1 with $m=1, n=2$, and obtain $\lambda_{0} \leq \max \left\{|\alpha|^{1 / 2},|\bar{\beta}|^{1 / 2}\right\}$. This result was essentially obtained by Liapounoff $[5 ; 6]$ in the study of $(4.1)$ with periodic $a(t)$.

For the case of periodic $a(t)$ many criteria are known to ensure that $\lambda_{0}=0$ (i.e. that all solutions of (4.1) remain bounded). See Liapounoff [6], Borg [3], and Bellman $[1 ; 2]$. If $a(t)$ is not periodic, but has a limit at infinity or other suitable properties, information on the precise asymptotic form of solutions of $(4.1)$ can be obtained. See Bellman, $[1 ; 2]$, Coddington and Levinson [4].

For certain applications to nonlinear theory it is of interest to know an upper bound on $\lambda_{0}$. In Theorem 2, we obtain an upper bound on $\lambda_{0}$ depending only on $\alpha$ and $\beta$, the bounds of $a(t)$.

TheOREM 2. Let a $(t)$ be real, piecewise continuous and bounded for all $t \geq 0$. For all $t \geq 0$, let $\alpha \leq a(t) \leq \beta$. Then for equation (4.1), $\lambda_{0} \leq \rho$, where $\rho$ is given by

$$
\begin{aligned}
& \rho=|\alpha|^{1 / 2} \text { if } 3 \alpha+\beta \leq 0, \\
& \rho=2^{-3 / 2}(\beta-\alpha)(\beta+\alpha)^{-1 / 2} \text { if } 3 \alpha+\beta \geq 0 .
\end{aligned}
$$

REMARK. If $a(t)$ is a nompositive constant, $\alpha=-A^{2}$, it is clear that $\lambda_{0}=A$. The theorem gives $\lambda_{0} \leq A$. If $a(t)$ is a positive constant, it is clear that $\lambda_{0}=0$. Here $\alpha=\beta$, so the theorem gives $\lambda_{0} \leq 0$. Thus for constant $a(t)$, the results are best possible.

Proof of Theorem 2. To prove the theorem we must show that if $\lambda>\rho$, and $x(t)$ is any nontrivial solution of (4.1), then both of $x(t) e^{-\lambda t}$ and $x^{\prime}(t) e^{-\lambda t}$ approach zero as $t$ becomes positively infinite.

Make the standard polar-coordinate transformation in (4.1). Setting $x=r \sin \theta, x^{\prime}=r \cos \theta$, we obtain the system

$$
\begin{aligned}
& r^{\prime}=r[1-a(t)] \sin \theta \cos \theta, \\
& \theta^{\prime}=\cos ^{2} \theta+a(t) \sin ^{2} \theta .
\end{aligned}
$$


For a nontrivial solution of (4.1), $r \neq 0$ for all $t$, and we assume $r>0$ for all $t \geq 0$.

For a nontrivial solution of (4.1), let $r_{0}$ be the value of $r$ at $t=0$. Define $\mu$ by

$$
\mu=\sup _{t \geq 1} \frac{1}{t} \log \frac{r}{r_{0}} .
$$

We assert that if $\lambda>\mu$, then both of $x(t) e^{-\lambda t}$ and $x^{\prime}(t) e^{-\lambda t}$ approach zero as $t$ becomes positively infinite. Since $r(t)^{2}=x(t)^{2}+x^{\prime}(t)^{2}$, it will be sufficient to show that $r(t) e^{-\lambda t}$ approaches zero. Since for $t \geq 1, r \leq r_{0} e^{\mu t}$, if $h=2^{-1}(\lambda-\mu)>0$, we have for $t \geq 1$

$$
r e^{-\lambda t}=r e^{-h t} e^{(h-\lambda) t}=r e^{-h t} e^{-(\lambda+\mu) t / 2} \leq r e^{-h t} e^{-\mu t} \leq r_{0} e^{-h t} .
$$

Hence $r e^{-\lambda t}$ approaches zero.

It is not difficult to obtain an upper bound for $\mu$. Indeed since

$$
\frac{1}{t} \log \frac{r}{r_{0}}=\frac{1}{t} \int_{0}^{t} \frac{r^{\prime}}{r} d t=\frac{1}{t} \int_{0}^{t}[1-a(s)] \sin \theta(s) \cos \theta(s) d s,
$$

and $|\sin \theta \cos \theta| \leq 1 / 2$, we have

$$
\frac{1}{t} \log \frac{r}{r_{0}} \leq \frac{1}{2 t} \int_{0}^{t}|1-a(s)| d s \leq \frac{1}{2} \sup _{t \geqq 0}|1-a(t)| .
$$

Hence

$$
\mu \leq \frac{1}{2} \sup _{t \geqq 0}|1-a(t)| .
$$

The final refinement of the estimate is made by making a change of independent variable $t=b \tau$, and selecting that value of $b$ giving the best estimate. If in (4.1) we set $t=b \tau,(b>0)$ and $x(b \tau)=y(\tau)$ we obtain

$$
\frac{d^{2} y}{d \tau^{2}}+b^{2} a(b \tau) y=0 \text {. }
$$

From the preceding we have that if $k>2^{-1} \sup \left|1-b^{2} a(b \tau)\right|, y e^{-k \tau}$ and $y^{\prime} e^{-k \tau}$ both approach zero. For such $k, x(b \tau) e^{-k r}$ and $x^{\prime}(b \tau) e^{-k \tau}$ both approach zero. Setting $b \tau=t$, and $k=b \lambda$, we find that $x(t) e^{-\lambda t}$ and $x^{\prime}(t) e^{-\lambda t}$ both approach zero if $\lambda>(1 / 2 b) \sup _{t \geq 0}\left|1-b^{2} a(t)\right|$.

We have shown so far that for any $b>0$, we have $\lambda_{0} \leq(1 / 2 b)$ sup $\left|1-b^{2} a(t)\right|$. We have $\alpha \leq a(t) \leq \beta$ for all $t \geq 0$.

If $\alpha+\beta \leq 0$, sup $\left|1-b^{2} a(t)\right|=1-\alpha b^{2}$. The minimum of $\left(1-\alpha b^{2}\right) /(2 b)$ occurs at $b^{2}=-1 / \alpha$, and is $(-\alpha)^{1 / 2}=|\alpha|^{1 / 2}$. If $\alpha=\beta=0, a(t) \equiv 0$, and $\lambda_{0}=0$. If 
$\alpha+\beta>0, \sup \left|1-b^{2} a(t)\right|=1-\alpha b^{2}$ if $b^{2} \leq 2 /(\alpha+\beta)$, but $=\beta b^{2}-1$ if $b^{2} \geq 2 /(\alpha+\beta)$. For $\alpha+\beta>0$, we then find

$$
\min _{b>0} \frac{1}{2 b} \sup _{t \geq 0}\left|1-b^{2} a(t)\right|
$$

occurs for $b^{2}=-1 / \alpha$ and equals $(-\alpha)^{1 / 2}$ if $3 \alpha+\beta \leq 0$, and occurs for $b^{2}$ $=2 /(\alpha+\beta)$ and equals $2^{-3 / 2}(\alpha+\beta)^{-1 / 2}(\beta-\alpha)$ if $3 \alpha+\beta \geq 0$.

From this last we see that $\lambda_{0} \leq \rho$, where $\rho$ is defined in the statement of the theorem. This completes the proof of Theorem 2 .

5. Application to damped systems. We now apply the result of Theorem 2 to the damped equation

$$
x^{\prime \prime}+c x^{\prime}+a(t) x=0 \quad(c>0) .
$$

We seek conditions on the damping constant $c$ which will ensure that all solutions of (5.1) approach zero as $t$ becomes positively infinite.

THEOREM 3. In equation (5.1) let $a(t)$ be positive, piecewise continuous, real and bounded for all $t \geq 0$. For all $t \geq 0$ let $0<\alpha \leq a(t) \leq \beta$. Then if $x(t)$ is any solution of (5.1), both $x(t)$ and $x^{\prime}(t)$ approach zero exponentially as $t$ becomes positively infinite whenever $c>\beta^{1 / 2}-\alpha^{1 / 2}$.

REMARKS. If $a(t)$ is a positive constant, the condition of the theorem is $c>0$, which is necessary and sufficient for the result.

Because of the behavior of (5.1) when $a(t)$ is a nonpositive constant, it is not possible to assert exponential decay of all solutions from a knowledge of merely the bounds of $a(t)$ when $a(t)$ is allowed to be nonpositive.

Proof of Theorem 3. In (5.1) set $x=y e^{-c t / 2}$. We obtain

$$
y^{\prime \prime}+\left(a(t)-\frac{c^{2}}{4}\right) y=0 \text {. }
$$

By Theorem 2, we can find an upper bound for $\lambda_{0}$ corresponding to (5.2). If we can show that $\lambda_{0}<c / 2$, the present theorem will be proved. Using Theorem 2 , we have $\lambda_{0} \leq \rho$, where

$$
\begin{array}{ll}
\rho=\left(\frac{c^{2}}{4}-\alpha\right)^{1 / 2} & \text { if } 3 \alpha+\beta \leq c^{2}, \\
\rho=2^{-3 / 2}(\beta-\alpha)\left(\beta+\alpha-\frac{1}{2} c^{2}\right)^{-1 / 2} \text { if } 3 \alpha+\beta \geq c^{2} .
\end{array}
$$

We shall show that if $c>\beta^{1 / 2}-\alpha^{1 / 2}$, then $\rho<c / 2$.

Since $\alpha>0$, if $c^{2} \geq 3 \alpha+\beta, \rho<c / 2$ from the first formula for $\rho$. If $c^{2}<3 \alpha+\beta$, the condition 


$$
2^{-3 / 2}(\beta-\alpha)\left(\beta+\alpha-\frac{1}{2} c^{2}\right)^{-1 / 2}<\frac{1}{2} c
$$

is found to reduce to

$$
\beta^{1 / 2}-\alpha^{1 / 2}<c<\beta^{1 / 2}+\alpha^{1 / 2} .
$$

But if $0<\alpha \leq \beta$, then $3 \alpha+\beta \leq\left(\beta^{1 / 2}+\alpha^{1 / 2}\right)^{2}$, so that when $c^{2}<3 \alpha+\beta$, the right hand condition is satisfied. Hence whenever $c>\beta^{1 / 2}-\alpha^{1 / 2}, \rho<c / 2$, and hence $\lambda_{0}<c / 2$. This completes the proof of Theorem 3 .

6. Application to Floquet theory. In the following we consider equation (4.1) with the additional assumption that $a(t)$ is periodic of period $L$. It is known from Floquet's theory that there exists a complex constant $\rho$, the real part of which is determined, such that the general solution of (4.1) can be written

$$
x(t)=C_{1} e^{\rho t} p_{1}(t)+C_{2} e^{-\rho t} p_{2}(t)
$$

where $p_{1}(t)$ and $p_{2}(t)$ have period $L$. If $\operatorname{Re} \rho \neq 0$, the imaginary part of $\rho$ is a multiple of $\pi / L$ as a consequence of $a(t)$ being real. A multiple of $2 \pi i / L$ may be added to $\rho$ without changing the form of the solution. If $\rho$ is a multiple of $2 \pi i / L$, the form of the solution is possibly

$$
x(t)=C_{1} p_{1}(t)+C_{2}\left[p_{2}(t)+t p_{1}(t)\right] .
$$

(When $a(t)$ is real it is possible to make $\rho$ real, but $p_{1}(t)$ and $p_{2}(t)$ are then possibly of period $2 L$.)

The solutions of (4.1) with $a(t)$ periodic are called stable if $\operatorname{Re} \rho=0$, and the exceptional case mentioned above does not occur. Conditions on $a(t)$ are known which ensure stability as was mentioned in $\$ 4$. From our point of view, $\lambda_{0}=\operatorname{Re} \rho$, so that bounds on growth of solutions with $a(t)$ periodic can be deduced from Theorem 2 .

THEOREM 4. In the differential equation

$$
x^{\prime \prime}+a(t) x=0,
$$

let $a(t)$ be real, piecewise continuous, periodic of period $L$, and let it satisfy $\alpha \leq a(t) \leq \beta$. Then if $\rho$ is the Floquet constant for solutions of (4.1),

$$
\begin{array}{ll}
\operatorname{Re} \rho \leq(-\alpha)^{1 / 2} & \text { if } 3 \alpha+\beta \leq 0, \\
\operatorname{Re} \rho \leq 2^{-3 / 2}(\beta-\alpha)(\beta+\alpha)^{-1 / 2} & \text { if } 3 \alpha+\beta \geq 0 .
\end{array}
$$

Proof of Theorem 4. Since $\operatorname{Re} \rho=\lambda_{0}$, this is an immediate corollary of Theorem 2.

When $\alpha=0$, Theorem 4 says that $\operatorname{Re} \rho \leq(\beta / 8)^{1 / 2}=0.35 \beta^{1 / 2}$. The bound of a constant times $\beta^{1 / 2}$ is the best possible type. The following is an example with $\alpha=0$ for which $\operatorname{Re} \rho=0.23 \beta^{1 / 2}$. 
Consider the equation $x^{\prime \prime}+a(t) x=0$, with $a(t)=\beta>0$ for $0 \leq t \leq \pi \beta^{-1 / 2} / 2$, $a(t)=0, \pi \beta^{-1 / 2} / 2<t<2 \pi \beta^{-1 / 2}=L$. If $\phi(t)$ is a solution such that $\phi(0)=1$, $\phi^{\prime}(0)=0$, and $\psi(t)$ is a solution such that $\psi(0)=0$ and $\psi^{\prime}(0)=1$, then it is known that $2 \cosh \rho L=\phi(L)+\psi^{\prime}(L)$. For the example at hand, $\phi(L)=-3 \pi / 2$, and $\psi^{\prime}(L)=0$. Thus

$$
\cosh \rho L=-3 \pi / 4, \text { so } \operatorname{Re} \rho=\frac{\beta^{1 / 2}}{2 \pi} \cosh ^{-1} \frac{3 \pi}{4}=0.23 \beta^{1 / 2} .
$$

\section{BIBLIOGRAPHY}

1. R. Bellman, A survey of the theory of the boundedness, stability, and asymptotic behavior of solutions of linear and nonlinear differential and difference equations, Office of Naval Research, Washington, 1949.

2. - Stability theory of differential equations, McGraw-Hill, 1953.

3. G. Borg, Über die Stabilität gewissen Klassen von linearen Differentialgleichungen, Arkiv för Matematik, Astronomi och Fysik vol. 31 no. 1 (1945).

4. E. A. Coddington and N. Levinson, Theory of ordinary differential equations, McGrawHill, 1955.

5. A. Liapounoff, Sur une série dans la théorie des équations différentielles lineaires du second ordre à coefficients périodiques, Zap. Imp. Akad. Nauk, Fiz.-Mat. Otd. vol. 13 no. 2 (1902).

6. - Problème général de la stabilité du mouvement, Annals of Mathematics Studies, No. 17, Princeton, 1949.

UNIVERSITY OF MiNNESOTA,

Minneafolis, MinN.

Massachusetts Institute of Technology,

Cambridge, Mass. 